\title{
ANTÁRTICA: DERRETIMIENTO DEL "WAIS" Y VOLCANISMO
}

\section{ANTARTIC: WAIS THAWING AND VOLCANISM}

\author{
Francisco Ferrando A.*
}

RESUMEN: El objetivo de esta investigación fue encontrar una explicación para la diferente y más rápida pérdida de masa del sector occidental de la Antártica o WAIS, lo cual se ha estado reflejando en desprendimientos masivos de los glaciares litorales, pérdida de espesor e incremento de la velocidad, lo cual en algunos de ellos ha alcanzado magnitudes no registradas hace miles de años (Domacket al., 2002). Más allá de los efectos del calentamiento global y del impacto humano directo, esta particularmente alta reducción del WAIS llevó a pensar en la concurrencia de otro factor genético, a saber, el volcanismo y el calor latente asociado en la base de la masa de hielo. Durante investigaciones llevadas a cabo en la Isla Rey Jorge, un fuerte retroceso y adelgazamiento de los glaciares fue detectado y cuantificado. Conjuntamente, varias evidencias de actividad volcánica pasada, como necks volcánicos, fragmentos de lava y piroclastos, fueron encontrados (Kellem et al., 2008; Ferrando et al., 2009). Estos hallazgos, junto a otras evidencias de volcanismo en el archipiélago de las islas Shetland del Sur y sus alrededores (Krauss, 2008; Ferrando et al., 2009), condujeron a realizar una investigación mediante el uso de imágenes satelitales en el sector de Mary Byrdland. Como resultado, diversas estructuras volcánicas fueron reconocidas en ese sector del WAIS. Consecuentemente, se llevó a cabo una búsqueda de bibliografía, encontrándose estudios desde los años 1993 y 1994 que hablan acerca de fuentes de calor bajo los glaciares. Posteriormente, en la primera década del siglo XXI, varios investigadores encontraron una gran cantidad de estructuras volcánicas, algunas de ellas activas en la Tierra de Mary Byrd y sus alrededores. Estas evidencias, y algunas otras como la existencia de glaciares rocosos en el sector de los valles secos de McMurdo (Bockheim, 2002), vienen a revelar una doble causa para el desigual nivel de consunción existente entre el WAIS y el sector oriental de la Antartica (EAIS). Adicionalmente, últimos estudios han establecido que el WAIS es el territorio más volcánico del planeta. Estos antecedentes no dejan duda acerca del rol del volcanismo en la mayor pérdida de hielo del WAIS respecto del EAIS, en la mayor pérdida de espesor y en la aceleración de los glaciares.

Palabras clave: WAIS, volcanismo, adelgazamiento del hielo, aumento de la velocidad de los glaciares. 
ABSTRACT: The objective of this research was to find the explanation for the different and faster loss of mass in the western sector of the Antarctic lce Sheet (WAIS), which has been reflected in massive coastal glaciers calving, thinning and increase in speed, which in some of them has reached magnitudes not recorded for thousands of years (Domack et al., 2002). Beyond the effects of the global warming and direct human impact, this particularly high reduction in WAIS led to the consideration of another genetic factor, namely volcanism and the associated latent heat at the base of the ice mass.

During research carried out on King George Island, a strong retreat and thinning of the glaciers was detected and quantified. Together, several evidences of past volcanic activity, such as volcanic necks, lava fragments and pyroclasts, were found (Kellemetals, 2008; Ferrando et al., 2009). These findings, together with other evidence of volcanism in and around the South Shetland archipelago (Krauss, 2008; Ferrando et al., 2009), led an investigation using satellite images in the Mary Byrd Land sector. As a result, several volcanic structures were recognized in that sector of the WAIS. Consequently, a literature search was carried out, finding studies since 1993 and 1994 that talk about heat sources under the glaciers. Subsequently, in the first decade of the 21 st century, several researchers found a large number of volcanic structures, some of them active in the Mary Byrd Land and its surroundings, and some others, such as the existence of rocky glaciers at the McMurdo dry valleys (Bockheim, 2002), reveal a double cause for the unequal level of consumption between WAIS and the eastern sector of Antarctica (EAIS). Additionally, recent studies have established that WAIS is the most volcanic territory on the planet. This background leaves no doubts about the role of volcanism in the greater loss of ice from WAIS than from EAIS, in the greater loss of thickness and in the acceleration of glaciers.

Key words: WAIS, volcanism, ice thinning, glaciers velocity increase.

Recibido: 1 1.03.19 Aceptado: 16.09.19

\section{INTRODUCCIÓN}

El proceso de calentamiento global que afecta al planeta ha estado influyendo negativamente en la criósfera como un todo, pero principalmente en el balance de masa de los inlandsis, campos de hielo y glaciares individuales (Vaughan, 2002; Turner, et al., 2005; Van den Broeke, 2005; Kellem, et al., 2010; Williams y Ferrigno, 2012; Bentley et al., 2014; Turner et al., 2016; Farinotti et al., 2019).

Ello se ha hecho más evidente a partir de los años 90's, en que a nivel atmosférico pasa gradualmente de la condición llamada Global Dimming a la de Global Brithening (Ferrando, 2013), implicando un incremento del "radiative forcing", es decir, de los watts/m2 que están llegando a la superficie de la Tierra y, por ende, a los cuerpos de hielo.

Todos estos fenómenos se han manifestado de forma diferencial en el continente antártico, hecho dado, por una parte, por sus características geológico-estructurales y topográficas. El continente antártico está conformado por dos secciones corticales de muy diferente magnitud. El sector oriental o EAIS (East Antarctic lce Sheet) y 
el sector occidental o WAIS (West Antarctic Ice Sheet). En su franja de contacto o sutura se encuentran los Antartandes o Andes Antárticos (Fig. 1).

Otro aspecto corresponde a las dimensiones areales y altitudinales del inlandsis, donde el EAIS representa aproximadamente el $80 \%$ de la superficie del continente antártico y altitudes máximas del orden de 4000 msnm, con espesores equivalentes de hielo o algo menores en su parte central dada la mayor altimetría del lecho rocoso, en buena parte sobre 750 msnm y localmente superando los 2250 msnm (Heinrich, 2008).

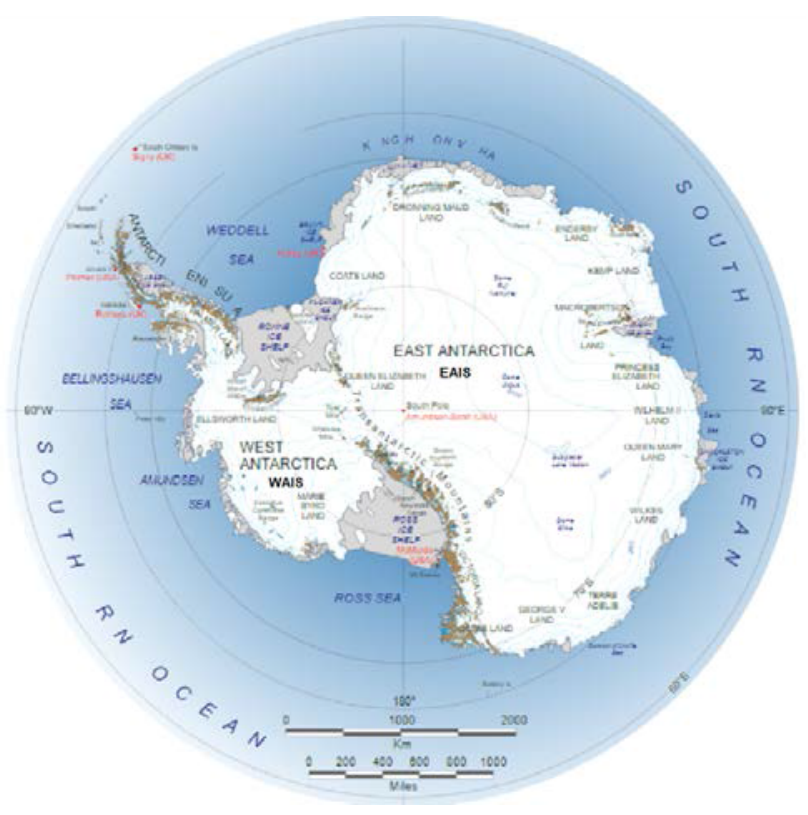

Figura 1. Las grades unidades glaciológicotopográficas del continente antártico.

Fuente: hitp://lima.nasa.gov/pdf/A3_overview.pdf

A diferencia, la sección occidental o WAIS, y sin considerar la Plataforma de Ross, representa del orden del 20\% de la superficie, y altitudes mucho menores asociadas a cordones montañosos y cumbres aisladas lentre 1800 y 2000 msnm), y espesores mucho menores de hielo. En este sentido, y dado que gran parte del lecho rocoso del WAIS se encuentra entre 0 y $750 \mathrm{~m}$ bajo el nivel del mar, y una parte relativamente menor entre 750 y 1500 m de profundidad Heinrich, 2008) los espesores de hielo variarían en promedio entre 2000 y 3000 m.

En este contexto, según Paolo et al. (2015), el WAIS ha presentado una mayor y persistente reducción de su volumen entre el año 1994 y el 2012 en relación al EAIS, cuya masa gélida presenta leves disminuciones solo a partir del año 2002. Esta situación del WAIS, además de las características generales señaladas, suele ser relacionada con el efecto del calentamiento global, pero esta no parece ser la única explicación.

Diversos antecedentes asociados a fuentes de calor endógeno comenzaron a surgir hace algunas décadas (Ej.: Payne, 1995) como causal coadyuvante del diferencial y acelerado derretimiento del sector occidental de la Antártica, tanto en la Península y sus alrededores como en los sectores de Ellsworth Land y Marie Byrd Land, hecho que está redundando en fenómenos de desprendimientos de megaicebergs y desintegración de plataformas de hielo como no se registraban hace miles de años (Domack et al., 2002).

Esto último está llevando a las lenguas de hielo que fluyen centrífugamente desde el interior del ice sheet antártico, y que alimentaban las plataformas de hielo flotantes, a carecer del freno u obstáculoque su masa implicaba. Consecuentemente, los glaciares están avanzando más rápido hacia el mar, donde son afectados por mareas, oleajes y corrientes (tidal glaciers) y sufren consecuentes desprendimientos (calvingl en sus frentes, liberando múltiples icebergs y contribuyendo al alzamiento del nivel medio de los océanos. 


\section{OBJETIVO}

Poner en evidencia la importancia y rol de la presencia de volcanismo en el mayor tenor de consunción del WAIS y, especialmente, en la aceleración de los glaciares alimentadores de las plataformas y el mayor desprendimiento de témpanos desde sus frentes.

\section{METODOLOGÍA}

A la luz de las investigaciones desarrolladas en glaciares individuales y lenguas efluentes del Campo de Hielos Krakovia en la Isla Rey Jorge, en el archipiélago de la Islas Shetland del Sur (Kellem et al., 2008; Ferrando et al., 2009), cuyos resultados indicaron fuertes retrocesos y adelgazamientos en décadas recientes, incremento de los días de lluvia respecto de los registros de precipitaciones sólidas, surgimiento y crecimiento de lagunas, cursos de agua y ambientes estivales claramente temperados, se procedió a buscar otros antecedentes que dieran cuenta del fuertemente negativo balance de masa de la criósfera de la isla, así como de las desintegraciones masivas, debilitamientos y macro-calving en el resto del WAIS, en contraste con un balance de masa claramente más equilibrado del EAIS.

Junto a los antecedentes de fuente primaria indicados, se buscó información sobre fuentes de calor endógeno y volcanismo en la antártica, partiendo por una interpretación y reconocimiento de formas que indicaran la presencia de volcanes en imágenes satelitales NOAA, U.S. Navy, NGA, GEBCO, PGC/NASA, USGS y MODIS. Identificadas diversas formas de impronta volcánica, se procedió a pesquisar y analizar información sobre volcanismo en la Antártica, así como de su relación con los cambios detectados en el WAIS.

\section{RESULTADOS}

El mayor calentamiento y la mayor ablación que se ha estado registrando desde décadas en los territorios englaciados al oeste de los Antartandes, hechos expresados en aceleraciones, adelgazamientos, desprendimientos y desintegraciones de masas de hielo, y la evidente diferencia con la mucho menor intensidad de estos fenómenos en el sector oriental o EAIS, tal como queda expresado en investigaciones llevadas cabo entre 1994 y 2012 (Paolo et al., 2015), llevó a plantearse la existencia de otra causa de estuviera contribuyendo esta desigual dinámica de consunción y reducción del volumen de hielo del sector occidental de la Antártica (Fig. 2).

Al respecto, King y Comiso (2003) y Turner et al (2005) reconocieron que las tendencias de las temperaturas en la Península Antártica no estaban sucediendo en ninguna otra parte de la Antártica, como tampoco en el resto del planeta (King y Harangozo, 1998; Marshall et al., 2002).

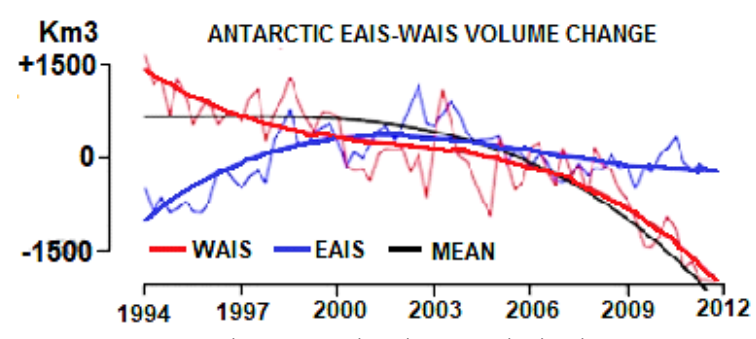

Figura 2. Cambios en el volumen de hielo entre el WAIS y el EAIS en el período 1994-2012. expresado en kilómetros cúbicos. (Paolo et al., 2015)

Las investigaciones de Kellem et al. (2008) y Ferrando et al. (2009) en glaciares como Wanda, Krack, Ecology, Znosco, Collins y Dobrowolski, ubicados en la Isla Rey Jorge (Shetland del Sur), junto al grado de redondeamiento de los detritos 
subglaciales y la presencia de flutes en los sectores liberados de hielo, indicadores de ambiente subglacial húmedo o politermal (Kellem et al., 2006; 2008), Ilevaron a evidenciar el efecto de calor latente proveniente del sustrato en el sector.

En cuanto a estructuras y manifestaciones extrusivas, se reconocen 10 centros volcánicos con actividad explosiva conocida o presumida durante el Cuaternario/Holoceno en el sector insular adyacente a la Península Antártica (Krauss, 2008). Entre los muchos antecedentes, están las islas volcánicas Deception, Penguin y Bridgeman, y formas remanentes como la Sail Rock (Krauss, 2008) y los neck's Ternick needle, Czajkowsky Needle y Arcktowsky Needle (Ferrando et al., 2009).
Además, desde el punto de vista litológico, el análisis de las rocas constitutivas de la Isla Rey Jorge (lavas y brechas) y los piroclastos encontrados con abundantes vesículas revelan su naturaleza volcánica y el registro de actividad explosiva en el pasado geológico (Ferrando et al., 2009).

Por otra parte, conocido es el sistema de microplacas como la Placa Drake, la Placa Scotia y la Placa de las Shetland del Sur, última que entra en contacto con la Placa Antártica en el Estrecho de Bransfield, donde se reconoce un adelgazamiento de la corteza y presencia de fallas y fracturas que posibilitan la salida de materiales magmáticos, dando origen a volcanes (Berrocoso et al., 2008) (Fig. 3).

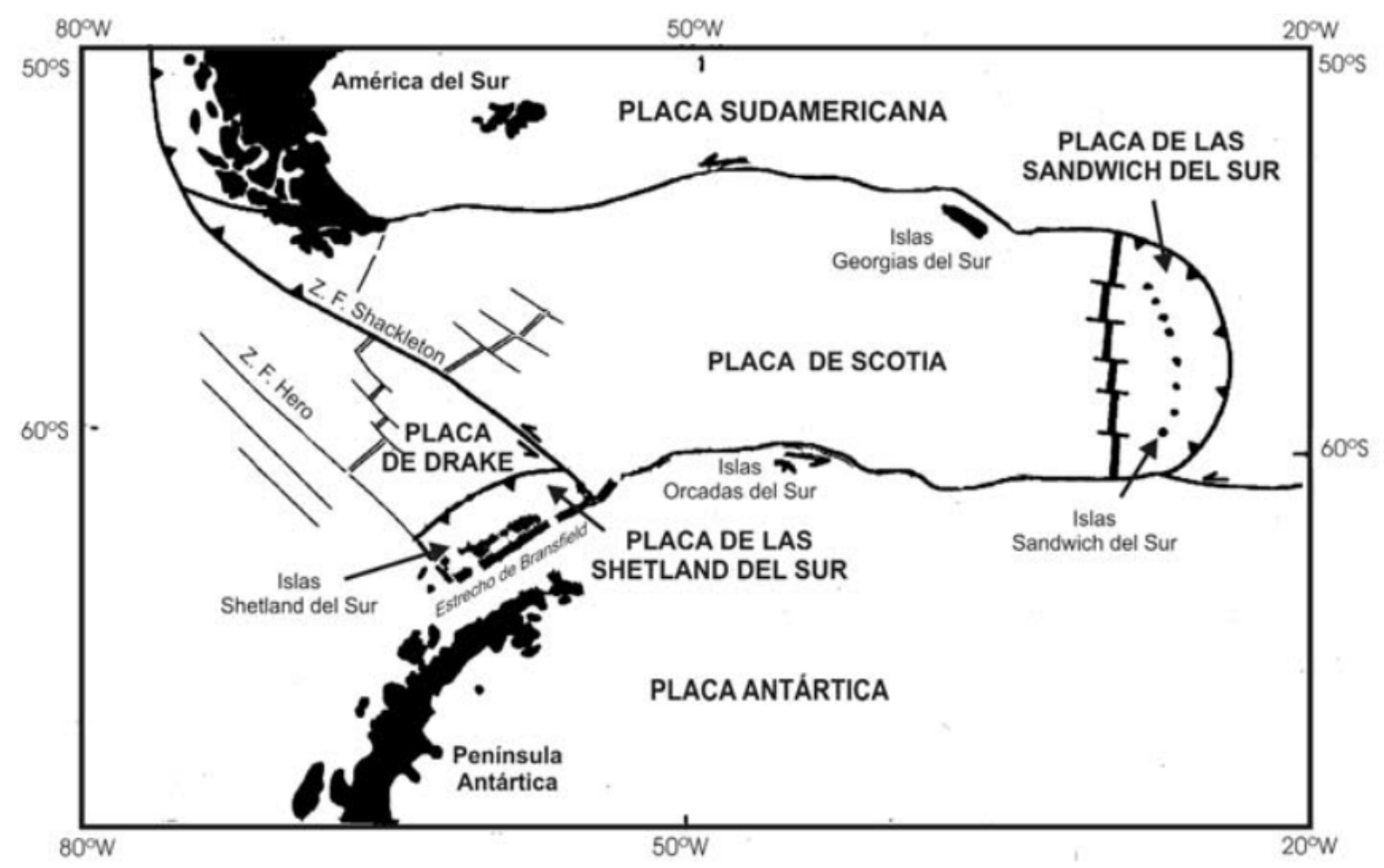

Figura 3. Sistema de placas del sector de las Islas Shetland del Sur. (Mod. de Berrocoso et al., 2008)

De acuerdo a este autor, se puede establecer que la ganancia y pérdida de energía termal por radiación desde el sustrato rocoso causa procesos que están directamente relacionados con la fusión de masas de hielo y nieve en su base, lo que causa adelgazamiento del ice sheet, de los glaciares y el incremento de flujos hídricos sub y supra glaciales. 
Ingresando en la tierra de Marie Byrd, la parte más ancha del WAIS, ya en el año 1963 Blankenship et al., señalaban la presencia de volcanismo activo y flujos de calor bajo el WAIS en base a evidencia aero-geofísica, lo que habría estado incrementando la fusión basal y el consecuente aceleramiento de las corrientes de hielo. Kintisch (2011) por su parte hace referencia a una masa de hielo gigante recongelada debajo del ice sheet Antártico, lo que revela momentos de fusión sub glacial.

En Pritchard et al. (2012) se reconoce la influencia de la fusión basal (basal melting)

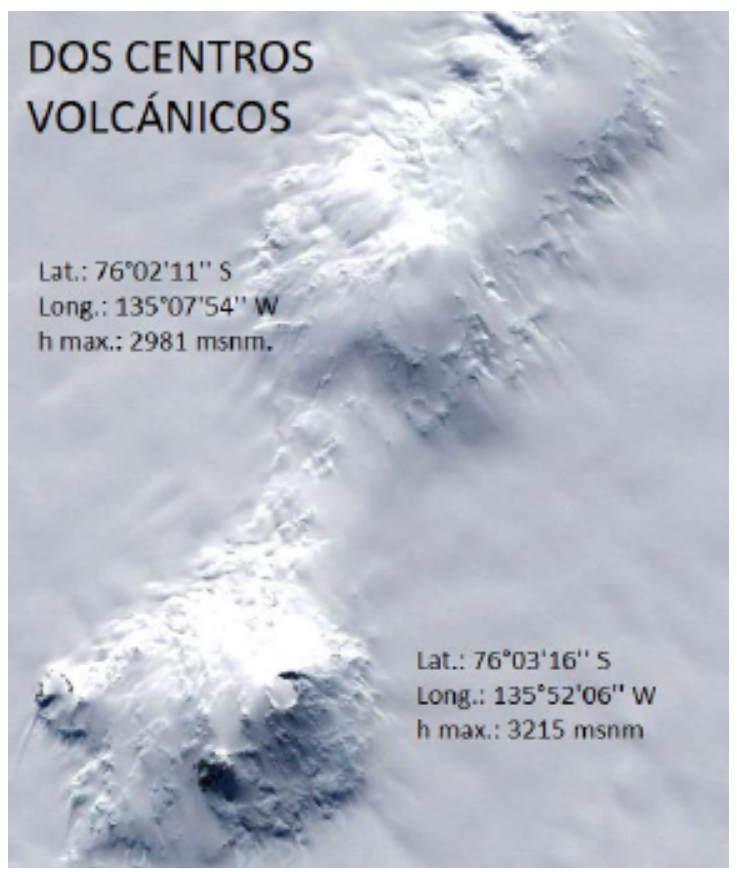

Figura 4. Imagen de 2 edificios volcánicos Mary Byrd Land (US Geological Service)

Ese mismo año, en van Wyk de Vries et al. (2017) se plantea si las amenazas al WAIS vienen desde abajo o desde arriba. Dicha investigación reconoce antecedentes de 47 volcanes de existencia conocida, como el Monte Erebus, a lo que se agregan otros 91, algunos de los cuales requieren de su comprobación, pues su existencia se basa como causa de la mayor velocidad de los flujos de hielo y plataformas como del incremento del adelgazamiento de ellas.

Luego, en el año 2013, Lough et al establecen la presencia de un nuevo volcán a $1 \mathrm{~km}$ bajo el hielo del WAIS con salida de magma que estaría generando millones de galones de agua, lubricando y acelerando el flujo de hielo.

En base en estos antecedentes, en un análisis de imágenes satelitales llevado a cabo el 2017, se reconoció la presencia de varias montañas claramente volcánicas en la tierra de Marie Byrd (Figuras 4 y 5).

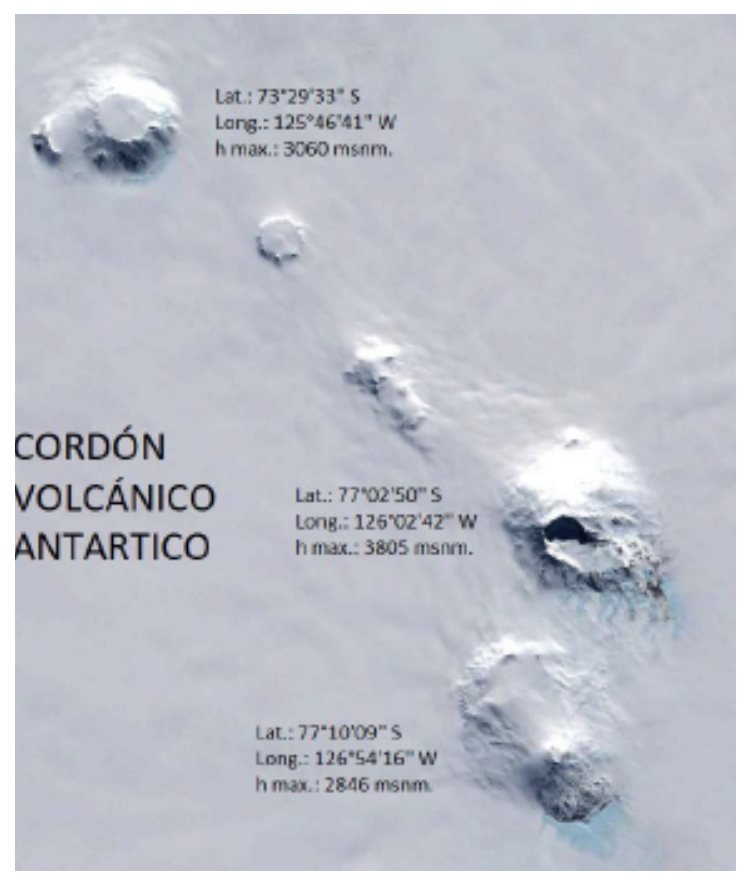

Figura 5. Imagen de cordón volcánico Mary Byrd Land (US Geological Service)

en perfiles geofísicos (magnetic \& gravity anomaly).

Contemporáneamente, Iverson et al. (2017) aportan al conocimiento con la primera evidencia física de volcanismo sub-WAIS. A ello se suma la existencia de valles secos (McMurdo Dry Valleys) y 
glaciares rocosos en Beacon Valley, lo cual no deja dudas respecto de la influencia del factor endógeno en el balance de masa negativo e inestabilidad del WAIS.

Un hecho indicativo y de gran trascendencia dice relación con la velocidad del Glaciar Pine Island y el fuerte calving que ha estado sufriendo su frente, como es el caso del reciente desprendimiento (Octubre/2018) de un fragmento de unos $300 \mathrm{~km} 2$. Esta conducta estaría asociada precisamente a la existencia de una fuente de calor volcánico en su lecho rocoso, lo cual fue descubierto recientemente en base a emanaciones de gases como helio-3, neón y xenón.

Acorde a lo anterior, van Wyk de Bries et al. (2017) plantea una nueva provincia volcánica y que el WAIS sobreyace al West Antarctic Volcánic Rift System (WAVRiS), el cual tendría más volcanes que el rift africano oriental.

\section{CONCLUSIONES}

Las investigaciones y análisis muestran inequívocamente que el WAIS está sufriendo una notable consunción en relación con la dinámica evolutiva del EAIS. Dicho proceso sería el resultado de efectos conjuntos del calentamiento global de la atmósfera, por encima, y de la ocurrencia de múltiples fuentes de calor endógeno, por debajo. Estas últimas incluyen volcanismo activo y calor latente, junto con el efecto sobre las aguas oceánicas sub-plataformas.

Estas fuentes de calor subglacial son consideradas responsables, en gran medida, tanto de las crecientes desintegraciones de las plataformascomo de la aceleración de los glaciares, cuyas lenguas alcanzan el litoral antártico (Tidal Glaciers) y el consecuente desprendimiento (calving) de icebergs de cientos de kilómetros cuadrados de extensión areal.

Si bien del total de volcanes del WAIS, de los cuales ya se conocían varios de ellos desde inicios de la década de los años 90's (Le Masurier et al., 1990; Siebert y Simkin, 2002), una parte importante de los recientemente detectados debe ser ratificada. Los análisis de los perfiles geofísicos del lecho subglacial, los que muestran su topografía, indican una alta probabilidad de que se trate de formas extrusivas.

Este proceso de consunción está ocasionando que, ante la desintegración - el desprendimiento de gigantescos fragmentos de algunas plataformas, se produzca una aceleración de los glaciares alimentadores de éstas, así como de otros glaciares (Ej: Pine Island Glacier), incrementándose el calving en magnitud y frecuencia. Es fundado plantear que este proceso, por tratarse de hielos que están sobre el continente antártico ly no flotando en dicho océano), estaría comenzando a influir con tendencia progresiva en el alzamiento del nivel del mar.

Adicionalmente, se considera plenamente justificado el que el Rift Antártico, por su gran cantidad de volcanes, sea considerado como continuación y parte del Cinturón de Fuego del Pacífico. 


\section{REFERENCIAS BIBLIOGRÁFIAS}

Bentley, M. J., Ó Cofaigh, C., Anderson, J.B., Conway, H., Davies, B., Grahan A.G.C., Hillenbrand, C.D., Hodgson, D.A., Jamieson S.S.R., Larter, R.D., Mckintosh, A., Smith, A.J., Verleyen, E., Ackert R.P., Bart, P.J., Brunstein, D., Canals, M., Colhoun, E.A., Crosta, X., Dickens, W.A., Domack, E., Dowdeswell, J.A., Dunbar, R., Ehrmann, W., Evans, J., Favier, V., Fink, D., Fogwill, C.J., Glasser, N.F., Gohl, K., Golledge, N.R., Goodwin, I., Gore, D.B., Greenwood, S.L., Hall, B.L., Hall, K., Hedding, D.W., Hein, A.S., Hocking, E.P., Jakobsson, M., Johnson, J.S., Jomelli, V., Jones, R.S., Klages, J.P., Kristoffersen, Y., Kuhn, G., Leventer, A., Licht, K., Lilly, K., Lindow, J., Livingstone, S.J., Massé, G., McGlone, M.S., McKay, R.M., Melles, M., Miura, H., Mulvaney, R., Nel, W., Nitsche, F.O., O’Brien, P.E.O., Post, A.L., Roberts, S.J., Saunders, K.S., Selkirk, P.M., Simms, A.R., Spiegel, C., Stolldorf, T.D., Sugden, D.E., van der Putten, N., Ommen, T., Verfaillie, D., Vyverman, W., Wagner, B., White, D.A., Witus, A.E., Zwartz, D (2014). A communitybased geological reconstruction of Antarctic Ice Sheet deglaciation since the Last Glacial Maximum. Quaternary Science reviews, $100,1-9$.

Berrocoso, M., Arias, M.R., Serrano, A., Fernández-Ros, A., de Gil, A., Torrecillas, C., García, J. A. (2008). Geodetic and thermometric monitoring of the volcanic activity at Deception Island (Antarctica): VOLDETEC Project (2005-2008). Libro de resúmenes del IV Simposio latinoamericano sobre investigaciones Antárticas y VII Reunión Chilena de investigación Antártica (Leppe, M., Gimpel, C., Leiva, L.F., eds), INACH, 382-3844

Bockheim, J. G. (2002). Landform and Soil Development in the McMurdo Dry Valleys,
Antarctica: A Regional Synthesis. Journal Arctic, Antarctic, and Alpine Research. 34(3), 308-317

Domack, E., Berger, G., Gilbert, R. (2002). Seafloor evidence of Larsen lce Sheet breackup, National Snow \& Ice data Center, USA. Recuperado de https://nsidc. org/news/newsroom/larsen_B/2002_ seafloor.html

Farinotti, D., Huss, M., Fürst, J.J., Landmann, J., Machguth, H., Maussion, F., Pandit, A (2019). A consensus estimate for the ice thickness distribution of all glaciers on Earth. Nature Geoscience, 12(3), 168-173.

Ferrando, F. J., Vieira, R., Kellem, K. (2009). El calentamiento global y la criósfera: Procesos y evidencias en el Glaciar Wanda, Isla Rey Jorge. Revista Investigaciones Geográficas 41, 25-40.

Ferrando, F. J. (2013). Cambio Climático: Del Global Dimming al Global Brigthening y la Evaporación en Chile. FAU-Universidad de Chile, 109.

Heinrich, P. (2008). Antarctic bedrock. jpg. Wikipedia. Recuperado de https://es.wikipedia.org/wiki/ Archivo:AntarcticBedrock.jpg

Iverson, N.A., Lieb-Lappen,R., Dunbar, N.W., Obbard, R. Kim, E., Golden, E. (2017). The first physical evidence of a subglacial volcanism under the West Antarctic Ice Sheet. Scientific Reports, 7, 1-8.

Kellem, K., Simoes, J. (2006). Dinâmica glacial e características sedimentares resultantes na zona proglacial da Geleira Ecology - Baía do Almirantado, Itha Rei George-Antártica. Revista Brasileira de Geomorfologia, 7(2), 51-60. 
Kellem, K., Ferrando, F.J., Simoes, J. (2008). Interpretación de la evolución del ambiente de deglaciación del Glaciar Wanda, Isla Rey George, Antártica. Libro de resúmenes del IV Simposio latinoamericano sobre investigaciones Antárticas y VII Reunión Chilena de investigación Antártica (Leppe, M., Gimpel, C., Leiva, L.F., eds), INACH, $261-264$.

Kellem, K., Vieira, R., Ferrando, F.A., Simoes, J.C (2010). Geomorfologia aplicada a reconstrução e ao monitoramento do impacto das mudanças climáticas em ambientes glaciais. Revista de Geografia, 27(1), 14.

King, J.C.; J.C. Comiso (2003) The spatial coherence of interannual temperatura variations in the Antarctic Peninsula. Geophysical Research Letter, 30(2), 1040.

King, J.C., Harangozo, S.A. (1998). Climate change in the western Antarctic Peninsula 1945-present: observations and possible causes. Annals of Glaciology, 27, 571-575

Kintisch, E. (2011). Gigantic remelted ice mass discovered below Antarctic lce Sheet. American Association for the Advancement of Sciencie, Recuperado de https://www.sciencemag.org/ news/2011/03/gigantic-remeltedice-mass-discovered-below-antarctic-icesheet

Kraus, S. (2008). Volcanismo explosivo de volcanes pleistocenos/ holocenos alrededor de la Península Antártica-Resultados de la campagna de terreno 07/08. Recuperado de https: / / www.researchgate. net/profile/Stefan_Kraus/ publication/200760533_Explosive_ volcanism_of_Pleistocene_Holocene_ volcanoes_around_the_northern Antarctic_Peninsula_-_Results_obtained_ during_the_field_campaign_0708/ links/0739f-7ea738443d 1 2acad57/ Explosive-volcanism-of-PleistoceneHolocene-volcanoes-around-the-northernAntarctic-Peninsula-Results-obtained-duringthe-field-campaign-07-08.pdf

LeMasurier, W.E., Thomson, J.W., Baker, P.E., Kyle, P.R., Smellie, J.L., Verwoerd, W.J. (eds.) (1990). Volcanoes of the Antarctic Plate and Southern Oceans. American Geophysical Union, 512.

Lough, A.C., Wiens, D.A., Bacheck, C.G., Anandakrishnan, S., Aster, R.C., Blankenship, D.D., Huerta, A.D., Nyblade, A., Young, D.A., Wilson, T.J. (2013). Seismic detection of an active subglacial magmatic complex in Marie Byrd Land, Antarctica. Nature Geoscience, 6, 1031 1035.

Marshall, G.J., Laguna, V., Lachlan-Cope, T.A. (2002). Change in Antarctic Peninsula tropospheric temperatures from 1956 to 1999; a synthesis of observations and reanalysis data. International Journal of Climatology, 22(3), 291-310.

Payne, A.J. (1995). Limit cycles in the basal thermal regime of ice sheets. Journal of Geophysical research, 100(B3), 42494263.

Paolo, F., Fricker, H., Padman, L. (2015). Volume loss from Antarctic ice shelves is accelerating, Science, 348162321, 327331. 
Pritchard, H.D., Ligtenberg, S.R.M. Fricker H.A., Vaughan, D.G., van den Broeke, M.R., L. Padman (2012). Antarctic icesheet loss driven by basal melting of ice shelves. Nature, 484, 502-505.

Siebert, L., Simkin, T. (2002). Volcanoes of the World: an Illustrated Catalog of Holocene Volcanoes and their Eruptions. Smithsonian Institution, National Museum of Natural History, Global Volcanism Program Digital Information Series GVP-3. Recuperado de https://avo.alaska.edu/ downloads/reference. php? citid=3261

Turner, J., Colwell, S.R., Marshall, G.J., Lachlan-Cope, T.A., Carleton, A.M., Jones, P.D., Lagun, V., Reid, P.A., lagorkina, S. (2005). Antarctic climate change during the last 50 years. International Journal of Climatology, 25, 279-294.

Turner, J., Lu, H., White, I., King, J. C., Phillips, T., Scott H., J, Bracegirdle, T. J., Marshall, G. J., Mulvane, R., Pranab D.
(2016). Absence of 21 st century warming on Antarctic Peninsula consistent with natural variability. Nature, 535, 41 1-415.

Van den Broeke, M. (2005). Strongsurface melting preceded collapse of Antarctic Peninsula ice shelf. Geophysical Research Letters, 32, 1-4.

Van Wyk de Vries, M., Bingham, R.G., Hein, A.S. (2017). A new volcanic province: an inventory of subglacial volcanoes in West Antarctic. Geological Society, 461, 18.

Vaughan, D.C., Spouge, J. R. (2002). Risk estimation of collapse of the west antarctic ice sheet. Climate Change, 52(1-2), 6591.

Williams, R.S., Ferrigno, J.G. (2012). State of the earth's cryosphere at the beginning of the 21 st century:glaciers, global snow cover, floating ice, and permafrost and periglacial environments, Professional Paper, 1386-A. 